\title{
Formation of young massive clusters from turbulent molecular clouds
}

\author{
Michiko S. Fujii ${ }^{1}$ and Simon Portegies Zwart ${ }^{2}$ \\ ${ }^{1}$ Division of Theoretical Astronomy, National Astronomical Observatory of Japan \\ 2-21-1 Osawa, Mitaka, Tokyo, Japan \\ email: michiko.fujii@nao.ac.jp \\ ${ }^{2}$ Leiden Observatory, Leiden University, Leiden 2300 RA, The Netherlands \\ email: spz@strw.leidenuniv.nl
}

\begin{abstract}
We simulate the formation and evolution of young star clusters from turbulent molecular clouds using smoothed-particle hydrodynamics and direct $N$-body methods. We find that the shape of the cluster mass function that originates from an individual molecular cloud is consistent with a Schechter function with power-law slopes of $\beta=-1.73$. The superposition of mass functions turn out to have a power-law slope of $<-2$. The mass of the most massive cluster formed from a single molecular cloud with mass $M_{\mathrm{g}}$ scales with $6.1 M_{\mathrm{g}}^{0.51}$. The molecular clouds that tend to form massive clusters are much denser than those typical found in the Milky Way. The velocity dispersion of such molecular clouds reaches $20 \mathrm{~km} \mathrm{~s}^{-1}$ and it is consistent with the relative velocity of the molecular clouds observed near NGC 3603 and Westerlund 2, for which a triggered star formation by cloud-cloud collisions is suggested.
\end{abstract}

Keywords. star clusters, young massive clusters, open clusters, numerical simulation

\section{Introduction}

Young massive clusters (YMCs) are more massive and much denser than typical open clusters (Portegies Zwart et al. 2010). In the Milky Way YMCs have been found in the last a few decades, but it remains unclear how they formed. In observed star forming regions the stars are spatially distributed in clumps or filaments, rather than in massive spherical distributions. We need to understand the link between such star forming regions and YMCs.

Numerical simulations of star cluster formation have been performed by several groups (e.g., Bonnell et al. 2003, 2008; Bate 2012; Federrath \& Klessen 2012; Federrath et al. 2014; Dale et al. 2014). These simulations include stellar growth using sink particles, and the residual gas is eventually removed by stellar feedback. Such numerical simulations are considered realistic, but because of the calculation cost the number of stars formed in such a simulation is limited to $\sim 10^{3}$.

We adopt a simpler approach in which the hydrodynamical simulations and N-body simulations are separated by assuming instantaneous star formation and gas expulsion at some point in the evolution (see Fujii \& Portegies Zwart 2015; Fujii 2015, for the details). This method is motivated by (Moeckel \& Bate 2010), and reduces the computational cost such that we can simulate up to $\sim 10^{5}$ stars. We use this method to perform a series of simulations to study how star clusters form from a variety of turbulent molecular cloud with a mass of $10^{4}$ to $10^{6} M_{\odot}$, and a density between 17 and $1700 \mathrm{~cm}^{-3}$. 


\section{Method}

We simulate star cluster formation in three steps:

1. hydrodynamical simulations of the collapse of a turbulent molecular cloud,

2. star formation based on the gas density distribution obtained from the hydrodynamical simulations, and

3. direct $N$-body simulations of stars based on the structure of the molecular clouds.

The hydrodynamical simulations are performed using the SPH code Fi (Hernquist \& Katz 1989; Gerritsen \& Icke 1997; Pelupessy et al. 2004; Pelupessy 2005), using the Astronomical Multipurpose Software Environment (AMUSE) (Portegies Zwart et al. 2013; Pelupessy et al. 2013). For initial conditions we adopt the isothermal homogeneous gas sphere, as was done in Bonnell et al. (2003), with a divergence-free random Gaussian velocity field $\delta \boldsymbol{v}$ with a power spectrum $|\delta v|^{2} \propto k^{-4}$ (Ostriker et al. 2001).

We adopt the total gas mass of $10^{4}$ to $10^{6} M_{\odot}$ and a mean density of $\sim 17$ to $\sim 1700 \mathrm{~cm}^{-3}$ (1 to $100 M_{\odot} \mathrm{pc}^{-3}$ ) assuming that the mean weight per particle is $2.33 m_{\mathrm{H}}$. As a consequence, the free-fall time $\left(t_{\mathrm{ff}}\right)$ of the gas cloud is $0.83,2.3$, and $8.3 \mathrm{Myr}$ for models with a density of 1,10 , and $100 M_{\odot} \mathrm{pc}^{-3}$, respectively. We set the gas temperature of $30 \mathrm{~K}$ and zero total energy (potential plus kinetic energy is zero). We adopt the mass of $\mathrm{SPH}$ particles of $1 M_{\odot}$, which is equal to the mean stellar mass of the following $N$-body simulations. The gravitational softening length is $0.1 \mathrm{pc}$. We set the number of particles within a smoothing length as 64 . With these settings, the smallest length scale we can resolve is $\sim 0.4$ pc.

After $0.9 t_{\mathrm{ff}}$, we stop the hydrodynamical simulations and analyze the density distribution of the collapsed molecular cloud. We replace some SPH particles with stellar particles by adopting the local star formation efficiency (SFE), $\epsilon_{\text {loc }}$, which depends on the local gas density $\rho$ given by $\epsilon_{\text {loc }}=\alpha_{\text {sfe }} \sqrt{\rho /\left(100 M_{\odot} \mathrm{pc}^{-3}\right)}$, where $\alpha_{\text {sfe }}$ is a coefficient which controls the star formation efficiency and a free parameter in our simulations, and we adopt $\alpha_{\text {sfe }}=0.02$. This SFE is motivated by the recent result that the star formation rate scales with free-fall time (Krumholz et al. 2012; Federrath 2013), and they obtained that the SFE per $t_{\mathrm{ff}}$ is 0.015 . With these settings, we obtain the global SFE for the entire regions of several per cent and the SFE of dense regions $\left(>1000 M_{\odot} \mathrm{cm}^{-3}\right)$ of $20-30$ $\%$, which is consistent with observations (Lada \& Lada 2003) and also simulations of molecular clouds with star formation (Federrath \& Klessen 2013).

After replacing gas particles with stellar particles, we remove all residual gas particles assuming it is expelled instantaneously, and we continue the simulation by means of direct $N$-body integration with stars only. We stop the simulation after $10 \mathrm{Myr}$. The $N$-body simulations were carried out with a sixth-order Hermite scheme (Nitadori \& Makino 2008) without any gravitational softening. We include stellar collisions by means of the sticky sphere approximation with stellar radius of zero-age main-sequence following the description of Hurley et al. (2000). We also include stellar mass-loss at the end of the main-sequence following Hurley et al. (2000) (see Fujii et al. 2009; Fujii \& Portegies Zwart 2013, for the details). We the a time step criterion of Nitadori \& Makino (2008) with an accuracy parameter, $\eta=0.1-0.25$. The energy error was smaller than $\sim 10^{-3}$ for all simulations over the entire duration of the simulations, which is sufficient to warrant an accurate representation of the simulation compared to a real star cluster Portegies Zwart \& Boekholt (2014). 

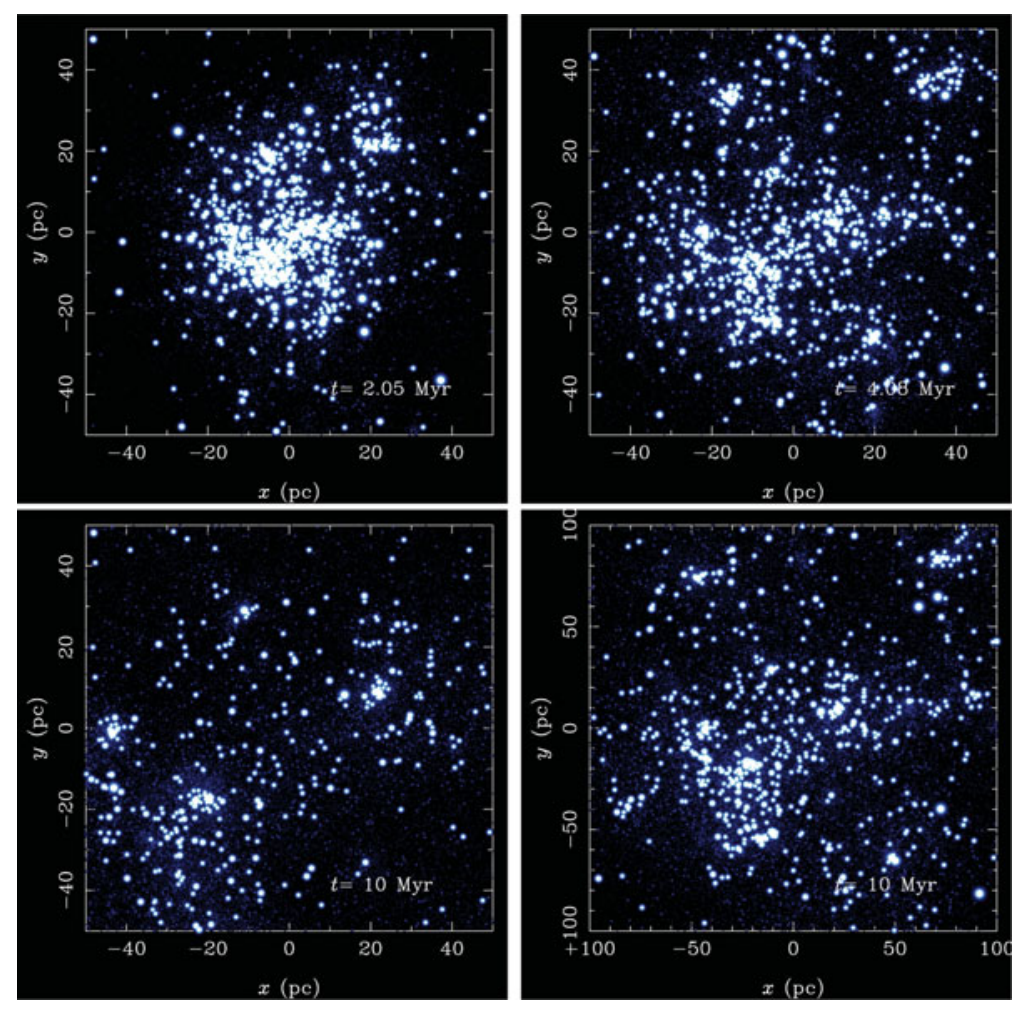

Figure 1. Snapshots of one of our model with a total gas mass of $10^{6} M_{\odot}$ and a mean density of $1700 \mathrm{~cm}^{-3}$. This model result in the formation of young massive clusters. The fractal dimension of this model is $\sim 1.4$ at $2 \mathrm{Myr}$.

\section{Formation of Young Massive Clusters}

In Figure 1, we present the stellar distribution that originate from a molecular cloud with a mass of $10^{6} M_{\odot}$ and an initial density of $\sim 1700 \mathrm{~cm}^{-3}$. These form the initial conditions for one of our $N$-body simulations. The time in this figure indicates the duration time after we stopped the hydrodynamical simulation and when we starting the $N$-body simulation. These initial condition are clumpy, which reflects the distribution of the gas. We recognize several tens of small stellar conglomerates, quite similar to observed stellar distribution of embedded clusters (Lada \& Lada 2003). At later time several of these clumps merge to more massive and larger clusters. Typically one or two clusters survive this hierarchical merger process and evolve to YMCs. This process is more clearly seen in our massive and dense initial conditions of the molecular clouds, those with a mass of $0.4-110^{6} M_{\odot}$ and a density of $100 M_{\odot}$. Such dense and massive molecular clouds typically appear in starburst galaxies, such as M83(Bastian et al. 2011) and M51(Chandar et al. 2011), but are absent in local disk galaxies such as the Milky Way (Krumholz et al. 2012) or M31.

There are only a few YMCs in the Milky Way, and therefore massive and dense molecular clouds must also form in the Milky Way. Recent observation of molecular clouds around YMCs in the Milky Way, such as NGC 3603 and Westerlund 2, suggested that they formed through collisions between giant molecular clouds (GMCs) with a velocity of $\sim 20 \mathrm{~km} \mathrm{~s}^{-1}$ (Furukawa et al. 2009; Ohama et al. 2010; Fukui et al. 2014). The velocity 

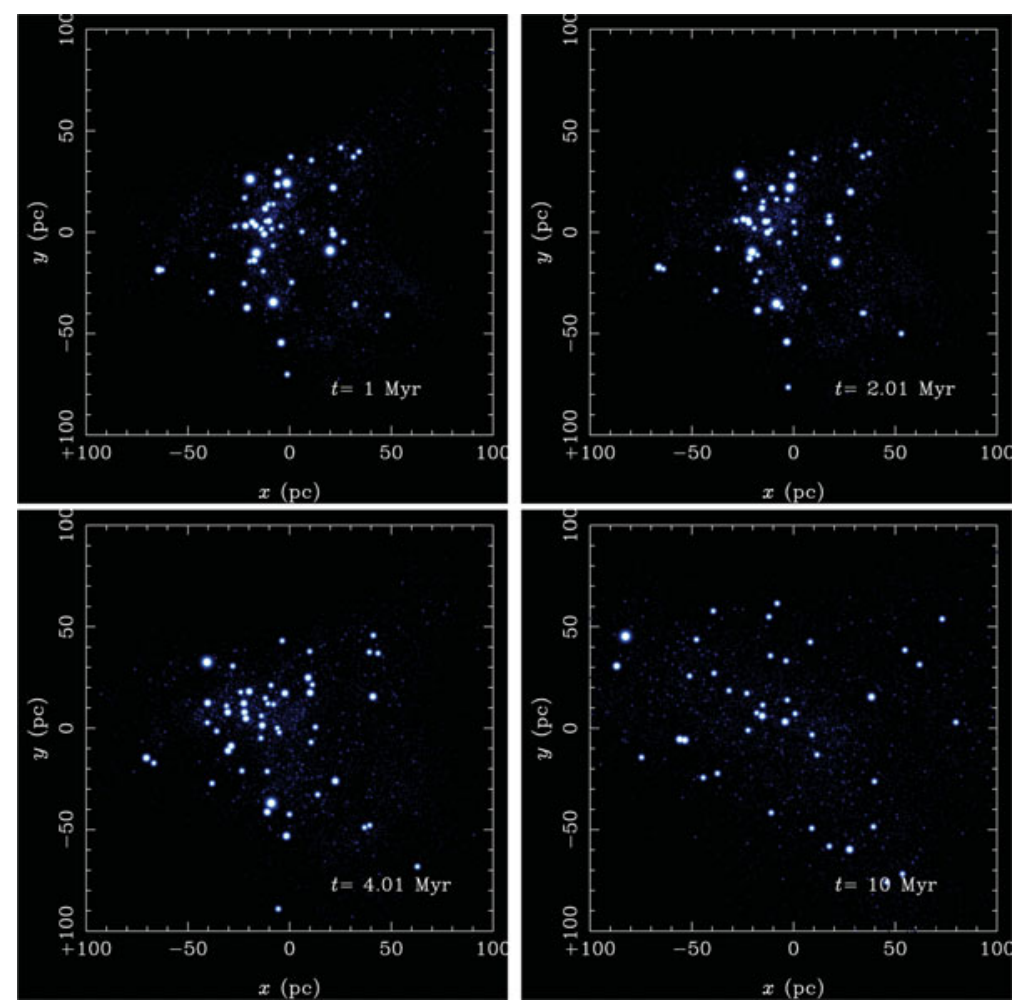

Figure 2. Snapshots of one of our model with a total gas mass of $10^{6} M_{\odot}$ and a mean density of $17 \mathrm{~cm}^{-3}$. This model form an association.

dispersion of our initial condition that form YMCs are consistent with these observed values.

Molecular clouds that typically appear in local disk galaxies, which follow Larson's relation Larson (1969), tend to evolve into open clusters and associations, rather than YMCs. In Figure 2, we present the snapshots of a model with a total gas mass of $10^{6} M_{\odot}$ but with a mean density of $17 \mathrm{~cm}^{-3}$. This molecular cloud forms fewer stars distributed in a more extended region. The final stellar distribution is less clumpy and does not contain any massive clusters, although some clumps appear very similar to classical open clusters. The distribution of stars from these simulations resembles OB associations, rather than star clusters.

\section{Mass Function of Star Clusters}

At $t=2$ and $10 \mathrm{Myr}$, we interrupt the simulations and search for clumps using HOP, (Eisenstein \& Hut 1998) in order to identify clusters. We fit a Schechter function (Schechter 1976):

$$
\phi(M) \equiv \frac{d N}{d M} \propto M^{\beta} \exp \left(-\frac{M}{M_{\text {cut }}}\right)
$$

to the resulting mass distribution. Here $M$ is the mass of clusters and $M_{\text {cut }}$ is the cut-off mass. Integration of equation (4.1) results in the cumulative mass function of the form

$$
N(>M) \propto M^{\beta+1} \exp \left(-\frac{M}{M_{\text {cut }}}\right) .
$$


For $M_{\text {cut }}$ we adopt the mass of the most massive cluster found in the simulations $\left(M_{\mathrm{c}, \max }\right)$. The best fit is achieved for a value for $\beta=-1.73$, which is consistent with the mass function for cluster observed in the Carina region (Feigelson et al. 2011). For $M_{\mathrm{c}, \max }$, we find the relation that $M_{\mathrm{c}, \max }=6.3 M_{\mathrm{g}}^{0.51}$. This is similar to the relation between the mass of the most massive star $\left(m_{\max }\right)$ and the mass of its host star cluster $\left(M_{\mathrm{c}}\right)$; $m_{\max } \simeq 0.4 m^{0.67}$ (Pflamm-Altenburg et al. 2007).

We superpose the fitted cluster mass function and obtain cluster mass functions for each disk galaxy. For the Milky Way we adopt a power of -1.45 (Planck Collaboration et al. 2011) and -0.9 for M31 (Kirk et al. 2013). We assume that each individual GMC forms a conglomerate of clusters that follow equation (4.2). The power-law index of the superposed cluster mass function is $\sim-2$ because of the cut-off in the Schechter mass function, which is consistent with cluster mass functions observed in nearby galaxies (Portegies Zwart et al. 2010).

\section{Summary}

We simulate the formation and evolution of young star clusters using SPH and direct $N$-body methods. We first perform SPH simulations of turbulent GMCs for one initial free-fall time, and analyze the resulting structure of the cloud. We subsequently replace a density-selected subset of SPH particles with stars by adopting a local star-formation efficiency that depends on the local gas density. The stellar distribution is clumpy and mimics known star forming regions.

We continue to evolve the stars dynamically by means of direct $N$-body integration and analyze the results at $2 \mathrm{Myr}$ and $10 \mathrm{Myr}$. The densest and most massive molecular clouds lead to the formation of YMCs via hierarchical merging of smaller clusters. The shape of the cluster mass function that originates from an individual molecular cloud is consistent with a Schechter function with a power-law slope of $\beta=-1.73$ at $2 \mathrm{Myr}$ and $\beta=-1.67$ at $10 \mathrm{Myr}$, which fits to observed cluster mass function of the Carina region. The superposition of mass functions have a power-law slope of $<-2$, which is consistent with the observed mass function of star clusters in the Milky Way, M31 and M83. We further find that the mass of the most massive cluster formed in a single molecular cloud with a mass of $M_{\mathrm{g}}$ scales with $6.1 M_{\mathrm{g}}^{0.51}$ which also agrees with recent observation in M51.

The molecular clouds which can form massive clusters are much denser than those typical found in the Milky Way. The velocity dispersion of such molecular clouds reaches $20 \mathrm{~km} \mathrm{~s}^{-1}$, which is consistent with the relative velocity of the molecular clouds observed near NGC 3603 and Westerlund 2. We argue that the formation of these clusters was initiated by the collision between molecular clouds.

\section{References}

Bastian, N., Adamo, A., Gieles, M., Lamers, H. J. G. L. M., Larsen, S. S., Silva-Villa, E., Smith, L. J., Kotulla, R., Konstantopoulos, I. S., Trancho, G., \& Zackrisson, E., 2011, MNRAS, 417, L6

Bate, M. R., 2012, MNRAS, 419, 3115

Bonnell, I. A., Bate, M. R., \& Vine, S. G., 2003, MNRAS, 343, 413

Bonnell, I. A., Clark, P., \& Bate, M. R., 2008, MNRAS, 389, 1556

Chandar, R., Whitmore, B. C., Calzetti, D., Di Nino, D., Kennicutt, R. C., Regan, M., \& Schinnerer, E., 2011, ApJ, 727, 88

Dale, J. E., Ngoumou, J., Ercolano, B., \& Bonnell, I. A., 2014, MNRAS, 442, 694

Eisenstein, D. J. \& Hut, P., 1998, ApJ, 498, 137 
Federrath, C., 2013, MNRAS, 436, 3167

Federrath, C. \& Klessen, R. S., 2012, ApJ, 761, 156

一, 2013, ApJ, 763, 51

Federrath, C., Schrön, M., Banerjee, R., \& Klessen, R. S., 2014, ApJ, 790, 128

Feigelson, E. D., Getman, K. V., Townsley, L. K., Broos, P. S., Povich, M. S., Garmire, G. P., King, R. R., Montmerle, T., Preibisch, T., Smith, N., Stassun, K. G., Wang, J., Wolk, S., \& Zinnecker, H., 2011, ApJs, 194, 9

Fujii, M., Iwasawa, M., Funato, Y., \& Makino, J., 2009, ApJ, 695, 1421

Fujii, M. S., 2015, PASJ, 67, 59

Fujii, M. S. \& Portegies Zwart, S., 2013, MNRAS, 430, 1018

一, 2015, MNRAS, 449, 726

Fukui, Y., Ohama, A., Hanaoka, N., Furukawa, N., Torii, K., Dawson, J. R., Mizuno, N., Hasegawa, K., Fukuda, T., Soga, S., Moribe, N., Kuroda, Y., Hayakawa, T., Kawamura, A., Kuwahara, T., Yamamoto, H., Okuda, T., Onishi, T., Maezawa, H., \& Mizuno, A., 2014, ApJ, 780, 36

Furukawa, N., Dawson, J. R., Ohama, A., Kawamura, A., Mizuno, N., Onishi, T., \& Fukui, Y., 2009, ApJl, 696, L115

Gerritsen, J. P. E. \& Icke, V., 1997, A\&A, 325, 972

Hernquist, L. \& Katz, N., 1989, ApJs, 70, 419

Hurley, J. R., Pols, O. R., \& Tout, C. A., 2000, MNRAS, 315, 543

Kirk, J. M., Gear, W. K., Fritz, J., Smith, M. W. L., Ford, G., Baes, M., Bendo, G. J., De Looze, I., Eales, S. A., Gentile, G., Gomez, H. L., Gordon, K., O’Halloran, B., Madden, S. C., Duval, J. R., Verstappen, J., Viaene, S., Boselli, A., Cooray, A., Lebouteiller, V., \& Spinoglio, L., 2013, ArXiv e-prints

Krumholz, M. R., Dekel, A., \& McKee, C. F., 2012, ApJ, 745, 69

Lada, C. J. \& Lada, E. A., 2003, AR\&A, 41, 57

Larson, R. B., 1969, MNRAS, 145, 271

Moeckel, N. \& Bate, M. R., 2010, MNRAS, 404, 721

Nitadori, K. \& Makino, J., 2008, New Astronomy, 13, 498

Ohama, A., Dawson, J. R., Furukawa, N., Kawamura, A., Moribe, N., Yamamoto, H., Okuda, T., Mizuno, N., Onishi, T., Maezawa, H., Minamidani, T., Mizuno, A., \& Fukui, Y., 2010, ApJ, 709, 975

Ostriker, E. C., Stone, J. M., \& Gammie, C. F., 2001, ApJ, 546, 980

Pelupessy, F. I., 2005, PhD thesis, Leiden Observatory, Leiden University, P.O. Box 9513, 2300 RA Leiden, The Netherlands

Pelupessy, F. I., van der Werf, P. P., \& Icke, V., 2004, A\& A, 422, 55

Pelupessy, F. I., van Elteren, A., de Vries, N., McMillan, S. L. W., Drost, N., \& Portegies Zwart, S. F., 2013, $A \& \& A, 557, \mathrm{~A} 84$

Pflamm-Altenburg, J., Weidner, C., \& Kroupa, P., 2007, ApJ, 671, 1550

Planck Collaboration, Ade, P. A. R., Aghanim, N., Arnaud, M., Ashdown, M., Aumont, J., Baccigalupi, C., Balbi, A., Banday, A. J., Barreiro, R. B., et al., 2011, A\& $A$, 536, A23

Portegies Zwart, S. \& Boekholt, T., 2014, ApJl, 785, L3

Portegies Zwart, S., McMillan, S. L. W., van Elteren, E., Pelupessy, I., \& de Vries, N., 2013, Computer Physics Communications, 183, 456

Portegies Zwart, S. F., McMillan, S. L. W., \& Gieles, M., 2010, AR\&A, 48, 431

Schechter, P., 1976, ApJ, 203, 297 